\title{
Mobilites Et Pratiques Socio-Spatiales Des Populations Des Zones Periurbaines De Daloa (Centre-Ouest De La Cote d'Ivoire)
}

\author{
Gohourou Florent \\ Mafou Kouassi Combo \\ Zadou Didié Armand \\ Université Jean Lorougnon Guédé, (Daloa, Côte d’Ivoire)
}

doi: 10.19044/esj.2017.v13n5p352 URL:http://dx.doi.org/10.19044/esj.2017.v13n5p352

\begin{abstract}
Currently, the urban spreading out is the core issue of all the territory management policy. In Côte d'Ivoire, it is particularly the main cause of over-mobility at the level of the displacements of proximity. This situation leads us to find out the real reasons for this generalized mobility among the concerned populations. Based on an inquiry strip, this study sets a broad map of the mobility and the spatial practices of the moving populations of four urban outskirts of Daloa: Bribouo, Sapia, Zaguiguia and Wandaguhé. The inquiry confirms the complexity and diversity that characterize the mobility practices by the so-called new-urban populations. On the one hand, the mobility of the urban outskirts populations are based on an unlimited number of factors such as: working life, social life, everyday life and depend on a large number of factors like: sex, residing duration, social status, community charges and finally differ from one individual to another. On the other hand, these populations are mainly in keeping with their movements on the scale of their villages and the whole city of Daloa.
\end{abstract}

Keywords: Spatial mobility, sociospatial practice, urban outskirts, urban spreading out, Daloa

\section{Resume}

Actuellement, l'étalement urbain est une problématique au cœur de toutes les réflexions politiques d'aménagement du territoire. En Côte d'Ivoire, il est notamment générateur de surmobilité au niveau des déplacements de proximité. Cette situation nous invite à nous enquérir des véritables facteurs de cette mobilité généralisée auprès des populations concernées. Reposant sur une enquête de terrain, cette étude dresse un tableau général des mobilités et des pratiques socio-spatiales des populations 
de quatre zones périurbaines en mouvement de Daloa: Bribouo, Sapia, Zaguiguia et Wandaguhé. L’enquête confirme la complexité et la diversité qui caractérisent les pratiques de mobilité des populations érigées en néocitadins à la faveur de l’étalement urbain. D’une part, les pratiques de mobilité des populations périurbaines se déclinent selon une infinité de moteurs (vie active, vie sociale, vie quotidienne), dépendent d'un grand nombre de facteurs (sexe, durée de résidence, statut social, responsabilité associative) et diffèrent finalement d'un individu à l'autre. D'autre part, ces populations inscrivent la majorité de leurs déplacements à l’échelle de leurs villages et de la ville de Daloa.

Mots-clés : Mobilité spatiale, pratique socio-spatiale, périurbain, étalement urbain, Daloa

\section{Introduction}

En Afrique subsaharienne, notamment en Côte d'Ivoire, « ces dernières décennies sont fortement marquées par une croissance urbaine rapide et un mouvement intense de périurbanisation. Ainsi, les villes se développent sur des périphéries de plus en plus lointaines » (Dziwonou, 2009). Cette dynamique spatiale favorise entre la ville et son hinterland des mouvements fréquents d'aller-retour incessants des populations (Alla, 1991). Aujourd'hui, l'ordre «mobilitaire » gagne donc du terrain ; les mobilités spatiales se développent, toujours plus nombreuses et plus lointaines, et nous invitent par ailleurs à réinventer notre rapport à l'espace et au territoire.

Le choix d'entreprendre une étude autour de la thématique des pratiques de mobilités des populations périurbaines s'inspire en grande partie de ces réflexions. Du point de vue des périurbains, on peut supposer que l'étalement urbain suscite bien davantage de bouleversement des repères spatiaux. C’est précisément l'objet de la réflexion que nous souhaitons mener ici : Comment l'urbanisation, par l'ensemble des mécanismes sociaux qu'elle induit, agit-elle sur les relations entre le périurbain et son espace ?

L’étude des relations hommes-espaces pose de nombreuses questions théoriques avec en premier lieu celle de convenir de la nature des manifestations concrètes de ces relations, et ce afin de définir une méthodologie opératoire. C'est vers toutes les formes de pratiques de l'espace que guide notre question de recherche, et en premier lieu vers l'analyse des mobilités spatiales. Celles-ci, parce qu'elles naissent des pratiques sociales des individus, nous sont naturellement apparues comme des objets d'étude.

L'entreprise d'une telle analyse nécessite un cadrage empirique restreint dans l'espace et limité d'un point de vue social. Elle réclame donc le choix d'un terrain et d'une population d'étude aux caractères opératoires 
dans notre problématique. Nous avons choisi d'entreprendre notre étude auprès des populations de quatre zones périurbaines en mouvement de Daloa : Sapia, Zaguiguia, Wandaguhé et Bribouo.

Notre travail sera organisé autour de trois axes. La première partie répondra à des questions simples: Que sous-tend l'investissement de l'espace par les périurbains de Daloa aujourd'hui ? Quelles pratiques de l'espace, quels types de mobilités développent-ils ? Quant à la deuxième partie, elle portera sur l'étude des individus selon leurs mobilités. Celles-ci au premier chef répondent à de multiples mobiles et diffèrent en fonction des individus et leurs particularités. La troisième et dernière partie de cette étude donnera à voir les échelles spatio-temporelles des pratiques spatiales des populations périurbaines de Daloa.

\section{Materiel et methode}

L'entreprise de cette étude a réclamé la construction d'une méthodologie inédite et complexe. En effet, l'essentiel des informations qui constituent le corpus de données de notre étude provient de questionnaires adressés directement à 200 personnes résidant dans quatre zones périurbaines en mouvement de Daloa : Sapia, Bribouo, Wandaguhé et Zaguiguia.

\section{Présentation de la zone de l'étude}

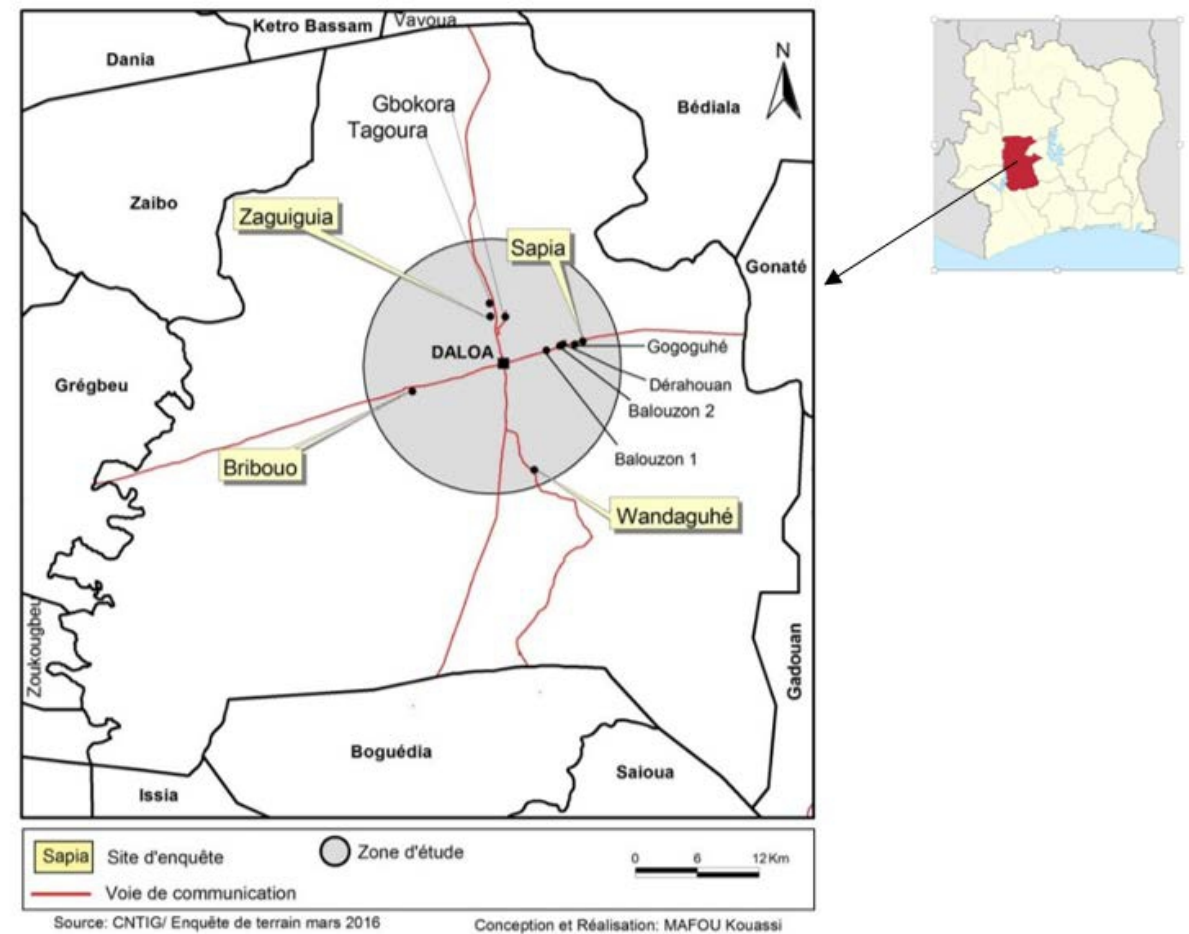


La composition du panel de ces personnes (une cinquantaine par site) dont les profils sociaux apparaissent dans les tableaux ci-dessous, s'est efforcée de comporter une répartition équilibrée de la situation familiale, de profils socio-professionnels, ainsi qu'une certaine diversité en termes de génération et de durée de résidence.

Tableaux récapitulatifs des caractéristiques des périurbains de Daloa enquêtés

\begin{tabular}{|c|c|c|c|c|c|}
\hline Localités & Bribouo & Sapia & Wandaguhé & Zaguiguia & $\begin{array}{c}\text { Total } \\
\text { général }\end{array}$ \\
\hline$[0-5$ ans [ & 1 & & & 8 & $\mathbf{1}$ \\
\hline$[10-15$ ans [ & 7 & 5 & 5 & 12 & $\mathbf{2 5}$ \\
\hline$[1-5$ ans [ & 8 & 6 & 7 & 5 & $\mathbf{3 3}$ \\
\hline$[15-20$ ans [ & 2 & 2 & 8 & 22 & $\mathbf{1 7}$ \\
\hline$[20$ ans et plus [ & 26 & 26 & 27 & 5 & $\mathbf{1 0 1}$ \\
\hline $\begin{array}{c}{[\text { Moins de } 1 \text { an }} \\
{[}\end{array}$ & 6 & 6 & 5 & 2 & $\mathbf{2 2}$ \\
\hline Total Général & $\mathbf{4 9}$ & $\mathbf{4 5}$ & $\mathbf{5 2}$ & $\mathbf{5 4}$ & $\mathbf{2 0 0}$ \\
\hline
\end{tabular}

Source : enquête de terrain, Mars 2016

Tableau 2 : Répartition par localité et par sexe des enquêtés

\begin{tabular}{|c|c|c|c|}
\hline Localités & Femmes & Hommes & Total général \\
\hline Bribouo & 24 & 25 & 49 \\
\hline Sapia & 15 & 30 & 45 \\
\hline Wandaguhé & 15 & 37 & 52 \\
\hline Zaguiguia & 19 & 35 & 54 \\
\hline Total Général & 73 & 127 & 200 \\
\hline
\end{tabular}

Source : enquête de terrain, Mars 2016

Tableau 3 : Répartition selon la localité et la situation matrimoniale

\begin{tabular}{|c|c|c|c|c|c|}
\hline \multirow{2}{*}{ Situation matrimoniale } & \multicolumn{5}{|c|}{ Localités } \\
\cline { 2 - 6 } & Bribouo & Sapia & Wandaguhé & Zaguiguia & Total général \\
\hline Célibataire & 19 & 6 & 6 & 16 & 46 \\
\hline Divorcé (e) & & & 1 & 1 & 2 \\
\hline Marié (e) & 25 & 7 & 36 & 12 & 81 \\
\hline Union libre & & 29 & 4 & 22 & 55 \\
\hline Veuf/Veuve & 5 & 3 & 5 & 3 & 16 \\
\hline Total général & 49 & 45 & 52 & 54 & 200 \\
\hline
\end{tabular}

Source : enquête de terrain, Mars 2016 
Tableau 4 : Répartition des enquêtés par localité et par profession

\begin{tabular}{|c|c|c|c|c|c|}
\hline Profession & Bribouo & Sapia & Wandaguhé & Zaguiguia & Total général \\
\hline Agent de sécurité & & & & 2 & 2 \\
\hline Aide-soignant (e) & & & & 1 & 1 \\
\hline Coiffeuse & & & & 1 & 1 \\
\hline Commerçant (e) & 11 & 2 & 2 & 7 & 22 \\
\hline Conducteur & & & & 2 & 2 \\
\hline Conseil Chefferie & & & & 1 & 1 \\
\hline Couturière & & & & 2 & 2 \\
\hline Cuisinière & & 1 & & & 1 \\
\hline Electricien & 1 & & & & 1 \\
\hline Elève & 1 & & & 3 & 4 \\
\hline Eleveur & 1 & & & & 1 \\
\hline Enseignant (e) & & 3 & 1 & 2 & 6 \\
\hline Etudiant & 2 & & & & 2 \\
\hline Footballeur & 1 & & & & 1 \\
\hline Gendarme & & 1 & & & 1 \\
\hline Maçon & & & & 2 & 2 \\
\hline Ménagère & 5 & 4 & & & 9 \\
\hline Menuisier & & & & 1 & 1 \\
\hline Militaire & & 1 & & & 1 \\
\hline Opérateur économique & & 2 & & & 2 \\
\hline Pasteur & 1 & & & & 1 \\
\hline Planteur & 25 & 5 & 42 & 15 & 87 \\
\hline Responsable ONG & & & & 1 & 1 \\
\hline Retraité & & 3 & 2 & 3 & 7 \\
\hline Sans emploi & 1 & 11 & 5 & 4 & 21 \\
\hline Technicien des TP & & 2 & & & 2 \\
\hline Transporteur & & & & 1 & 1 \\
\hline Sans précision & 1 & 10 & & 6 & 17 \\
\hline Total Général & 49 & 45 & 52 & 54 & 200 \\
\hline
\end{tabular}

Source : enquête de terrain, Mars 2016

Le choix de ces périurbains s'est opéré par hasard, au fil des rencontres, et par interconnaissance. Quant aux remplissages des questionnaires, ils se sont déroulés, en majorité, chez les personnes enquêtées elles-mêmes, et dans une moindre mesure, dans des lieux " publics » (restaurants, buvettes « maquis » notamment). Les questionnaires avaient pour objectif d'identifier concrètement les pratiques sociales et spatiales de ces populations, à l'aide de questions précises sur leurs activités et les déplacements qu'elles suscitent. 
Nous avons par ailleurs effectué des entretiens de groupe avec trois entités par village. Ces entretiens ne se sont pas déroulés sur la base des questions du questionnaire. Cependant, Ils nous ont permis de collecter des informations précieuses et complémentaires. Nous nous sommes ainsi entretenus avec les associations des jeunes, la chefferie villageoise et l'association des femmes afin d'avoir une idée accrue et enrichie du profil global des périurbains de Daloa, de leur situation socio-économique, de leur vie sociale et culturelle. Nous avons pu ainsi obtenir des informations intéressantes sur l'investissement associatif de ces périurbains et son sens, mais aussi sur le recours ou la participation de ces derniers aux structures locales.

Un dernier procédé nous est apparu riche d'informations et de résultats, celui de l'observation et de la discussion informelle. Il s'agissait, lors de moments précis (marché hebdomadaire, festivités locales, fêtes associatives,...) et dans des lieux spécifiques (école primaire, terrain de football transport en commun,...) d'observer en quantité (durée, fréquence,...) et en qualité (où va-t-on ? Avec qui ?...) les déplacements des zones périurbaines.

Nous avons choisi d'accompagner cette méthode d'un autre mode d'investigation. Il s'agit du parcours dans le village. Il consiste, dans la mesure du possible, à faire une "sortie » avec le périurbain interrogé afin qu'il nous montre les lieux appréciés et appropriés, les lieux antipathiques et peu fréquentés ; il nous en explique en outre ses motivations.

Le parcours vient confirmer ou infirmer les résultats des techniques précédentes. Il nous permet la visualisation concrète de l'espace de vie du périurbain, leurs itinéraires privilégiés.

Le principal inconvénient de ce procédé a été le temps et l'investissement qu'il demandait pour le périurbain ; pour cette raison, nous n’avons pu en réaliser que quelques-uns, qui se sont cependant avérés des moments sympathiques et privilégiés de dialogues et d'échanges.

\section{Resultats et discussions}

\section{Sources de l'investissement de l'espace par les periurbains}

Après avoir décrit les périurbains à travers leur spécificité, notre propos s'inscrit dans cette partie dans l'exploration de leurs pratiques sociospatiales. Celles-ci se déclinent autour de facteurs multiples et selon des réalités très diverses. L'espace est finalement très inégalement utilisé selon les individus et leurs caractéristiques. De l'analyse apparaissent un certain nombre d'éléments déterminants des formes de pratiques spatiales des populations périurbaines. 


\section{La vie active (domicile-travail et déplacement domicile-étude)}

L'activité professionnelle $(36 \%)^{15}$ constitue parallèlement aux activités académiques (20\%) une source première des déplacements des périurbains. Il s’agit autant des mobilités spatiales directes entreprises pour se rendre sur le lieu de travail ou de formation, que des déplacements secondaires effectués dans le cadre de l'activité principale (recherche d'emploi, activité syndicale, fréquentation des bibliothèques,...).

Quelles que soient les dimensions prises par ces déplacements, le travail et les études sont des occasions de déplacements journaliers ou quasiquotidiens pour 56\% des périurbains enquêtés. L’espace du travail, de l'activité scolaire ou universitaire représente de ce fait, pour la plupart de ceux avec lesquels nous nous sommes entretenus, celui où ils passent la plus grande partie de leur temps et le lieu d'étude du travail ou de formation représente un pôle central de leur espace de vie.

Dans le cas des périurbains qui sont sans emploi $(21 \%)^{16}$, la recherche d'un emploi constitue un moteur de mobilité majeur, mais leur mobilité de travail se caractérise davantage par l’irrégularité.

Finalement, à l'image des conclusions établies par X. Piolle (1979), auprès des habitants de Pau ou celle d'A. Frémont (1978) auprès des habitants de Caen, le rôle éminent du travail dans la mobilité des périurbains enquêtés ne se dément que rarement.

\section{La vie sociale : visites aux amis, parents et voisins}

Comme chez la plupart des Ivoiriens, les activités de sociabilité occupent une place importante dans la vie des populations enquêtées. Après l'intérêt accordé au travail ou à la formation, c'est surtout la famille, les relations amicales et les relations associatives qui sont les moteurs principaux qui permettent de compléter la compréhension des comportements spatiaux des périurbains.

Leurs relations familiales sont d'abord des frères et sœurs ${ }^{17}$, mais aussi des cousins; donc des relations de génération. Quant aux autres relations familiales, elles ne sont pas négligeables puisque $61 \%$ rendent très souvent visites à leurs oncles et tantes qui résident dans le même village qu'eux.

\footnotetext{
15 36\% des personnes enquêtées effectuent quotidiennement des déplacements de travail ; soit $12 \%$ à Zaguiguia, $2 \%$ à Wandaguhé, $13 \%$ à Sapia et $9 \%$ à Bribouo.

${ }^{16} 21 \%$ des périurbains effectuent des navettes dans le cadre de leur recherche d'emploi : $1 \%$ à Bribouo, $11 \%$ à Sapia, à $5 \%$ à Wandaguhé et $4 \%$ à Zaguiguia. $7 \%$ des personnes enquêtées sont des tiers absents ; soit à $3 \%$ à Sapia, $1 \%$ à Wandaguhé et $3 \%$ à Zaguiguia.

${ }^{17} 90 \%$ des personnes enquêtées ont déclaré avoir au moins un parent ou proche dans le village.
} 
Dans les zones périurbaines, la présence au village d’un oncle est essentielle, car il assure une fonction d'accueil ou d'assistance à leurs neveux et nièces. L’oncle le plus âgé au village joue le rôle de père de familles. En revanche, c'est aux oncles et tantes paternelles que l'on rend le plus souvent visite.

Pour les femmes, la belle famille est quasiment plus importante que la famille. Par conséquent, elles rendent plus souvent visite à leurs belles familles qu’à leur propre famille.

Quand des frères et sœurs sont séparés, ils se rendent aussi visite régulièrement mais seulement quand ils ont des affinités ou quand un frère plus âgé dirige les plus jeunes.

La visite à la famille est donc un aspect caractéristique des populations des zones périurbaines. C'est une pratique sociétale (et pour les plus jeunes souvent une obligation sociale) profonde et la distribution spatiale des membres de la famille dans l'espace rural, urbain, national ou international est facteur de connaissance de l'espace par ces populations.

D’une autre forme, mais pouvant aussi être un facteur de la mobilité des périurbains, les relations amicales permettent aussi une connaissance de l'espace. Car, les amis sont souvent éloignés les uns des autres. Ils habitent soit dans un autre village soit dans la ville de Daloa. Les amis se rencontrent le plus souvent dans le domaine scolaire ou universitaire, à l'église, dans les buvettes «maquis » et restaurants, aux réunions associatives, mais très peu dans les domiciles. En outre, de ces initiatives privées ou parfois familiales, ces populations sont loin de rester en retrait des diverses formes de la vie associative. En effet, 12\% des périurbains enquêtés ont déclaré avoir milité activement ou passivement dans des mouvements associatifs locaux. Qu'elles soient sportives, religieuses, récréatives, politiques, ou une association des originaires d'une région ou d'un pays, elles sont toutes sources de nombreux déplacements. C'est surtout quand les réunions sont fixées dans des espaces éloignés qu’elles occasionnent une bonne connaissance de l'espace parcouru lors du déplacement.

\section{La vie quotidienne : achats, religion, démarches...}

Les périurbains enquêtés n’échappent pas aux contingences de la modernité, qui les amènent à des déplacements très fréquents, et ce hors des déplacements d'étude, de sociabilité ou de travail. De nombreuses autres activités sont autant d'opportunité de mouvement, à l'exemple de toutes les autres activités domestiques, administratives, médicales...

Selon les individus, ces activités peuvent occuper une proportion très importante dans les mobilités régulières. L’activité d’achat et d'approvisionnement domestique représentent ainsi un moteur de mobilité important pour les périurbains rencontrés. A l’image de Frémont (1978) qui 
avait montré que le lieu d'achat constituait pour les Caennais un des trois principaux foyers de leur espace de vie, notre enquête montre aussi que les déplacements liés à l'activité de chalandise sont parmi les motifs de déplacement les plus cités chez les périurbains de Daloa.

Nous nous sommes aussi interrogés sur l'existence de moteurs de déplacement propres aux autochtones des zones périurbaines. Dans notre cas, les recherches que nous avons menées ne nous en indiquent pas. Il semble en fait que les moteurs de mobilité soient les mêmes pour les autochtones que pour les allogènes, mais ces moteurs agissent avec une intensité différente.

L’activité universitaire ou le travail fonctionne comme le moteur premier de mobilité dans tous les cas, mais les activités de sociabilité occupent une place privilégiée pour les autochtones. L’enquête témoigne, en outre, du peu de temps et de déplacement consacré par ces derniers à des loisirs individuels. Si le sport a pu être ponctuellement évoqué, la promenade, les activités ludiques (en famille ou entre amis) ou les vacances sont assez peu mentionnées comme génératrices d'une mobilité géographique.

\section{Les periurbains ; une pratique spatiale differenciee : l'effet de quelques composantes individuelles}

La mobilité géographique des périurbains enquêtés répond à de multiples mobiles et diffère selon les individus et leurs particularités. Selon qu'il soit homme ou femme, actif ou non, autochtone ou non, les espaces sociaux se nuancent, les raisons de se déplacer varient et contribuent ainsi à la construction d'espaces de vie différenciés.

\section{La durée de résidence}

Puisqu'elle influence obligatoirement les pratiques sociétales, mais aussi parce que la connaissance de l'espace en dépend, la durée d’installation peut se définir comme un premier facteur discriminant des comportements spatiaux des périurbains. En effet, « On ne connaît pas le même espace selon que l'on est en ville depuis longtemps ou que l'on vient d'y arriver, que l'on vit depuis des années dans le même quartier » (Lambony, 1994).

Les périurbains nouvellement installés, à l'exemple de ceux que nous avons pu enquêter, se déplacent essentiellement à l'échelle de la ville de Daloa. La solitude qu’ils ressentent dans les zones périurbaines entraîne une participation très assidue aux réunions d'association d'originaire et une fréquentation régulière des aires de loisirs (bars, maquis, terrains de football) dans la ville de Daloa même lorsque les ressources financières sont très modestes, comme le cas des élèves périurbains enquêtés. Souvent, quand les ressources financières le permettent, les vacances sont pour eux l'occasion 
d’un retour auprès des parents généralement installés dans une ville de la région du Haut-Sassandra au Centre-ouest de la Côte d’Ivoire.

\section{Sexe}

Etre homme ou femme ne semble pas constituer en soi un facteur majeur dans la détermination de la fréquence ou de l'ampleur des déplacements et la division sexuelle de l'espace semble davantage régner dans les rôles et dans les différents lieux fréquentés par les périurbains enquêtés. Dans la majorité des cas, les espaces publics tels que les lieux dédiés au sport, les maquis sont davantage fréquentés par les hommes alors que les femmes pratiquent moins ces espaces publics et leurs déplacements s’organisent plus autour des tâches ménagères et des soins aux enfants. Le rare temps libre est passé à l'église le dimanche, avec des amies dans la même situation, ou est utilisé pour rendre visite à leur famille dans le village ou dans la ville de Daloa.

\section{Statut social}

Bien que dans les zones périurbaines, les populations constituent majoritairement une population en activité, le statut social a été identifié comme un facteur qui sous-entend des comportements spatiaux différenciés. Ainsi, en dehors des trajectoires pour se rendre quotidiennement sur le lieu de travail ou dans les activités extrascolaires (loisirs, services administratifs, visites, etc.), les déplacements pour la recherche d'emploi font partie du quotidien de certains périurbains enquêtés. Ce type de mobilités organise l'espace-temps de cette catégorie de périurbains. Les étudiants périurbains effectuent aussi des déplacements de recherche d'emploi mais seulement si la période des examens est éloignée, et si les horaires du travail proposés leur conviennent parfaitement, ou bien enfin s'ils n’ont pas une contrainte académique (stage obligatoire dans le cursus de formation notamment).

\section{Le pouvoir discriminant de la responsabilité associative}

Nous avons montré dans ce travail, la place de prédilection de la sociabilité familiale et communautaire dans les pratiques spatiales des périurbains. Qu'elle soit une visite amicale et familiale, ou une participation à toutes formes d'entraides africaines (cotisation pour envoie d'un corps dans le village d’origine, présenter les condoléances à un ami,...), aux activités (associatives ou non) coordonnées par les périurbains ou par un membre éloigné de leur famille (fêtes religieuses, baptême, mariage,...), elles participent de manière très importante à leurs mobilités et encore plus particulièrement à celles de leurs représentants associatifs. En effet, que l'on soit simple adhérent ou responsable et actif, l'espace de vie du périurbain varie. Le fait d’être un simple membre ou de figurer dans le bureau de 
l'association est une source de déplacements à l'échelle locale, alors qu'occuper une fonction décisionnelle est génératrice d'une mobilité plus soutenue et déclinée parfois à une autre échelle que le local. Ce sont les membres du bureau de l'association qui incarnent naturellement les villageois devant les autorités locales. A cet effet, leur comparution sur la scène publique, les sollicitations dont ils sont l'objet, au cours des événements socioculturels du village, les poussent à des déplacements plus réguliers et à la fréquentation d'un certain nombre de structures étatiques (Mairie, Préfecture).

\section{Les echelles spatio-temporelles de la mobilite des populations periurbaines}

Après avoir analysé les moteurs de la mobilité géographique des périurbains et les facteurs discriminants de leurs comportements spatiaux, notre réflexion s'oriente maintenant vers les espaces qu'ils pratiquent et connaissent de même que, vers les lieux structurants de leurs espaces de vie. Même si aujourd'hui, les moteurs de leurs déplacements sont complexes, force est de reconnaître que certaines constantes se retrouvent dans les pratiques spatiales des périurbains: le local occupe majoritairement une position centrale, une place de prédilection dans leurs espaces de vie, et les autres échelles (régionale, nationale, et internationale) sont des territoires des basses fréquences, des déplacements rares voire: mensuels, annuels ou strictement occasionnels.

\section{Les zones périurbaines et la ville de Daloa: lieux des pratiques quotidiennes}

Alors que les formes de mobilités géographiques se diversifient, que le local est de moins en moins l'échelle pertinente pour en analyser les composantes (Knafou R. et al, 1998), l'enquête montre que les périurbains inscrivent la majorité de leurs déplacements à l'échelle locale et microlocale.

\section{La sur-importance des "mobilités sédentaires", inscrites dans la régularité et la localité}

Les observations menées auprès des périurbains témoignent de leur attachement au territoire local. Ce repli qui transparaît quasiment dans leur vie quotidienne est à lier à la satisfaction de leurs besoins premiers à l'échelle de la ville de Daloa voire même des villages périphériques (Zaguiguia, Bribouo, Wandaguhé, Sapia) et ses quartiers.

Cependant, selon le village ou le quartier de résidence, leurs pratiques spatiales changent. Ainsi, les habitants des villages de Sapia et Zaguiguia entretiennent plus de relations régulières avec leurs villages et ses 
équipements. A contrario, les périurbains qui résident dans les villages (Wandaguhé et Bribouo) qui n’ont pas une gamme assez complète d’équipements se trouvent dans l'obligation d'étendre la majorité de leurs mobilités quotidiennes dans la ville de Daloa.

Ces différents constats qu'ils soient tirés de l'enquête par questionnaire ou des entretiens menés auprès des périurbains convergent donc dans la mise en évidence d'un certain nombre de réalités :

Les activités professionnelles et académiques qui s’exercent toujours dans la ville de Daloa ou dans les zones périurbaines (Wandaguhé, Bribouo, Zaguiguia, Sapia) suscitent des mobilités quotidiennes qui ont la particularité d’être généralement très intenses. La localisation excentrée de l'Université Jean Lorougnon Guédé (UJLoG) et de certains établissements scolaires ou grandes écoles privées entraînent le développement de navettes, très polarisées. Les élèves et étudiants qui ne séjournent pas dans les territoires qui abritent ces établissements publics ou privés se déplacent quotidiennement pour aller dans leurs lieux de formation. Les déplacements des périurbains dans l'espace local et à Daloa n’est pas exclusivement liés aux activités professionnelles et académiques. Les nécessités de leur vie quotidienne entrainent d'autres types de déplacements, liés à d'autres facteurs. L'achat des besoins domestiques premiers se fait dans les boutiques qui prolifèrent à l’échelle de Daloa ou des villages périphériques (Zaguiguia, Bribouo, Wandaguhé, Sapia). Les périurbains qui résident dans les villages moins équipés administrativement ou domestiquement se trouvent dans l'obligation d'étendre leurs déplacements vers la ville de Daloa naturellement mieux équipées.

Parfois, les équipements publics, les familles, les édifices religieux, les établissements bancaires,... présents dans la ville de Daloa comme l'église catholique, le stade de football de Daloa, l’hôpital général de Daloa induisent une mobilité relativement intense et une distribution spatiale plus complexe. Ainsi, certains périurbains qui résident dans le village de Sapia se rendent une à deux fois par semaine à l'église catholique du centre-ville de Daloa pour célébrer la messe du dimanche. D’autres périurbains qui résident dans les villages excentrés de Wandaguhé et Bribouo rendent visite deux à trois fois par semaine à leurs tantes respectivement dans les villages périphériques de Zaguiguia et Sapia.

Autres phénomènes qui témoignent aussi de la quotidienneté des mouvements géographiques des villageois dans les zones périurbaines sont les activités de loisirs et les mouvements associatifs. Même si les activités de loisirs (football, bars, restaurations,...) peuvent se faire localement, 64\% des périurbains enquêtés fréquentent très souvent d'autres territoires pour se distraire loin des regards parentaux : «Je me déplace quasiment tous les week-ends en dehors du village avec mes amis pour partir consommer de la 
bière dans les buvettes de Daloa pour échapper au regard de mes parents. », confie un étudiant de Sapia. Enfin, les activités associatives qui mobilisent notamment la chefferie se déroulent au domicile du chef du village ou dans le centre-ville, devant la Mairie ou la Préfecture. On peut ainsi voir les membres du bureau de la chefferie devant l'hôtel de ville ou la Préfecture de police pour venir défendre les intérêts de leur village.

\section{Le centre-ville de Daloa : un point fort de l'espace de vie des périurbains}

De l'enquête, on a pu déduire qu'il se dégage dans l'espace de vie des périurbains un point fort dont la pratique est partagée par la quasi-totalité des personnes enquêtées. Ce point est le centre-ville de Daloa, c'est-à-dire le quartier commerce.

Pour comprendre le pouvoir attractif de cet espace sur les périurbains, il n'est pas inutile de noter que c'est le lieu de localisation de toutes les fonctions administratives qui leurs sont utiles. C'est toujours là que tout converge. C'est à cet endroit que se concentrent, la Mairie, la Préfecture, l'administration fiscale,...et avec bien entendu, la grande majorité des établissements bancaires pour les questions de prestige.

A côté de l'implantation de ces établissements qui peut être considérée comme l'élément moteur de la convergence des périurbains dans le centre-ville, c'est aussi le lieu d'indiquer que les déplacements des périurbains ont d'autres ressorts que les contraintes administratives. Il reste que ce centre-ville est aussi le lieu de prédilections de la grande majorité des manifestations sociales auxquelles ils ont été conviés dans les zones périurbaines.

\section{Autres échelles d'espace, autres échelles de temps : l'étalement des distances et l'allongement des fréquences}

Au-delà de la zone périurbaine et de la ville de Daloa, les mouvements géographiques des périurbains sont très inégaux et soumis à de multiples moteurs. Une composante s'en dégage néanmoins : la rareté des déplacements.

\section{Arythmie et indigence des pratiques de l'espace régional}

En considérant tous les déplacements qu'effectuent les périurbains enquêtés vers les villes de la région du Haut-Sassandra (exceptée Daloa), quel qu'en soit le moteur, on peut mettre en évidence un schéma particulier. Ils entretiennent des relations très timides avec le reste de la région et sa périphérie. Des distinctions remarquables apparaissent toutefois entre les périurbains enquêtés.

On notera que l'espace régional est parfois fréquenté par 15\% des périurbains enquêtés

(les originaires des autres villes de la 
région) c’est-à-dire la catégorie des périurbains qui s’est trouvée dans l'obligation de s’implanter dans la ville de Daloa à la faveur notamment de la crise politico-militaire ou pour y exercer une activité professionnelle ou universitaire (Onusiens, fonctionnaires, étudiants...). Dans ce cas, les périurbains effectuent parfois des visites à leur ville d’origine. La fréquence des visites est à mettre en rapport avec l'éloignement de la région de résidence des parents ou de la famille du périurbain : elle peut atteindre 3 fois par an pour une ville située à proximité de Daloa. Cependant, elle n’est que d'une fois par an ou tous les deux ans pour les localités très éloignées comme Issia, Vavoua et Zoukougbeu.

On note aussi que le statut d'autochtone du village (Wandaguhé, Bribouo, Zaguiguia ou Sapia) se révèle une variable très discriminante pour la mobilité des périurbains vers les autres villes de la région du HautSassandra. Ici, contrairement à la première catégorie de périurbains, hormis les déplacements exceptionnels (funérailles, fêtes de générations, baptême,...) dans les autres villages de la région, c'est l'absence de motivation, d'un « rapport social sous-jacent » (Begag, 1991), qui est en cause. La famille déjà présente dans la zone périurbaine exclue de fait toutes visites familiales en dehors de Daloa ou de la zone périurbaine. En outre, les périurbains ne pratiquent quasiment pas de loisirs qui les conduisent en dehors de Daloa.

\section{La place privilégiée d'Abidjan dans les mobilités des périurbains à l'échelle nationale}

En ce qui concerne les déplacements nationaux, les tendances sont similaires à celles observées précédemment, où, avec l’augmentation de la distance de déplacement. La mobilité décroît quel que soit le motif. L'absence de motifs explique encore une fois la rareté des déplacements à cette l'échelle.

Pour les périurbains qui effectuent ces mobilités, les motifs familial, amical, professionnel et administratif, constituent majoritairement la première cause des déplacements. Ainsi, quand le calendrier s’y prête, la visite à un parent, à un ami ou le renouvellement d'un extrait de naissance, l'exécution d'un stage de fin d'études, mais aussi la recherche insatisfaite à l'échelle locale d'un travail peut requérir leur présence dans certaines villes. Les périurbains avec lesquels nous nous sommes entretenus, se rendent notamment à San Pedro, Bouaké, pour des visites familiales et même à Korhogo pour un stage comme c'est le cas d'un étudiant enquêté à Bribouo.

Abidjan apparaît malgré tout comme une destination privilégiée par rapport à la place qu'occupent les autres villes ivoiriennes dans les destinations des périurbains. Si les visites familiales conduisent une à deux fois par an les périurbains dans les villes comme Bouaké, Agnibilékrou, 
Grand-Bassam et Divo, le poids de ce motif est très important dans le départ vers la capitale économique Abidjan. En effet, lorsqu’on observe la mobilité à l'échelle nationale des périurbains, l’on s'aperçoit que $53 \%$ d'entre eux ont effectué deux à trois fois par an une visite de courtoisie à Abidjan. Il faut aussi ajouter que l’afflux vers la capitale est sans doute motivé par le prestige de la ville elle-même et sa représentation en Côte d'Ivoire, voire en Afrique. C’est pourquoi, malgré la proximité relative dans l'espace de certains centres urbains comme Bouaflé et Yamoussoukro, les étudiants périurbains préfèrent faire leurs stages à Abidjan.

\section{L'échelle internationale : une échelle moins connue}

Dans un contexte où les flux de personnes traversent encore aujourd'hui l'Afrique et singulièrement sa partie occidentale via notamment les guerres civiles avec leur cortège de réfugiés, la migration des personnes quittant principalement les zones rurales des pays enclavés, ou à faible revenu, comme la Guinée, le Burkina, le Mali en direction des pays relativement développés (Côte d’Ivoire, Sénégal), il ressort que les périurbains enquêtés sont de véritables " chanceux » ou des " sédentaires » dans leur village de résidence. En effet, sur l'ensemble des personnes enquêtées seulement 2 burkinabé et 1 malien ont déclaré avoir déjà effectué une visite familiale dans leur pays d'origine respectif. Pour le reste de notre population d'étude, c'est encore une fois, l'absence de revenu et de motivation qui sont en cause. Ils sont bien épargnés par les nombreuses guerres civiles que connaissent encore aujourd'hui les Etats ouest africains. L'absence de moyen financier et de parents très proches dans le pays d'origine ne suscitent pas d'engouement particulier pour les déplacements internationaux.

\section{Conclusion}

La description des mobilités et des pratiques socio-spatiales des périurbains de Daloa témoigne de leur complexité et de leur diversité. Elles se déclinent selon une infinité de moteurs, qui dépendent d'un grand nombre de facteurs et différent finalement d'un individu à l'autre. En règle générale, l'activité professionnelle, académique, la sociabilité et la vie domestique sont les moteurs de leurs mobilités. Cependant, de l'analyse de certaines variables comme la durée de résidence, le sexe, le statut social et la responsabilité associative, il ressort que leur pratique spatiale varie selon les individus. Un certain nombre de traits apparait en outre récurrents dans la construction des espaces de vie de ces périurbains : l'hyperlocalité de la majorité des formes de mobilités et l'existence de centres de gravité collectifs situés autour du quartier commerce de Daloa et de la ville d'Abidjan, puis la rareté des déplacements aux échelles nationale et internationale. 


\section{References:}

1. Alla D A (1991), Dynamique de l'espace péri-urbain de Daloa, Thèse de Doctorat de $3^{\text {ème }}$ cycle, Géo, Université d’Abidjan, 313p.

2. Bailly A. (1986), «La perception de la ville et déplacements. L’impact de la mobilité sur le comportement », Cahier de géographie du Québec, vol.18, n 45, p. 525-540.

3. Begag A. (1991), La ville des autres. La famille immigrée et l'espace urbain, Lyon, Presse Universitaires de Lyon

4. Clément C. (2000), Espace de vie, espace en ville: Parcours migratoires, représentations et pratiques de l'espace urbain à Niamey. Lyon : Université de Lyon 2, Faculté de sciences économiques et de Gestion, 354p. Th. doc. : Eco : Lyon.

5. Di Méo G. (1987), « Les formations socio-spatiales », Annales de géographique, n537, p. 564-594.

6. Dziwonou Y. (2009), Etalement urbain et les difficultés de mobilité dans les métropoles d'Afrique subsaharienne, Revue de Géographie Tropicale et d'Environnement, n² 2, 2009, pp. 60-75.

7. Frémont A. (1978), Espace et cadre de vie : l'espace de vie des caennais, Caen, Université de Caen et Ministère de la qualité de la vie, CERA.

8. Gohourou F., (2013), « Migrations internationales et territorialisations : les Français dans la ville d'Abidjan (Côte d’Ivoire), Thèse de Doctorat, Géo. Université de Poitiers, 382 p.

9. Haumont A. (1993), «La mobilité intra-urbaine : rétrospective et perspective », Les Annales de la recherche urbaine, $n^{\circ} 59-60$, juinsept., p.109-118.

10. Knafou R. et al. (1998), La planète nomade, les mobilités géographiques aujourd'hui, Paris éd. Belin, coll. Les Atlas de l'Afrique.

11. Lambony G-P. (1994), De Lomé à Harare, le fait citadin : images et pratiques des villes africaines, Paris, éd. Karthala, coll. Hommes et Sociétés.

12. Levy J-P, Dureau F (2002), L'accès à la ville : les mobilités spatiales en questions, Paris, éd. L’Harmattan, coll. Habitat et sociétés.

13. Piolle X. (1979), les citadins et leur ville, paris, éd. des sciences de l'homme, Privat

14. Sidikou H A. (1974), Sédentarité et mobilité entre Niger et Zgaret, Paris, éd. Copedith, coll. Etudes nigériennes

15. Thumerelle P-J. (1986), Peuples en mouvement : la mobilité spatiale des populations, Paris, éd. SEDES-CDU, coll. DIEM, Dossiers des images économiques du monde. 